\title{
Supplemental Online Documents
}

Ann Fam Med 2010;8(Suppl 1):s92. doi:10.1370/am.1144.

Context for Understanding the National Demonstration Project and the Patient-Centered Medical Home

Kurt C. Stange, William L. Miller, Paul A. Nutting; Benjamin F. Crabtree, Elizabetb E. Stewart; Carlos Roberto Jaén

Supplemental Appendix. Summary of the Joint Statement of 4 Physician Organizations on

Principles of the Patient-Centered Medical Home

http://www.annfammed.org/cgi/content/full/8/suppl_1/s2/DC1

Methods for Evaluating Practice Change Toward a Patient-Centered Medical Home

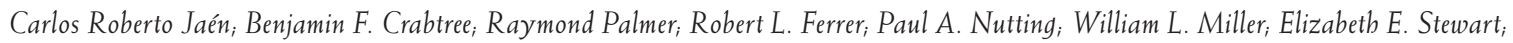
Robert Wood; Marivel Davila, Kurt C. Stange

Supplemental Appendix 1. Baseline Practice Survey

Supplemental Appendix 2. Chart Review

Supplemental Appendix 3. Depression Measures

Supplemental Appendix 4. Clinician Staff Questionnaire

Supplemental Appendix 5. Financial Survey

Supplemental Appendix 6. Practice Environment Checklist

Supplemental Appendix 7. TransforMED Key Stakeholder Interview Guide

Supplemental Appendix 8. TransforMED Practice Interview Guide

Supplemental Appendix 9. NDP Evaluation Team Site Visit Guide

Supplemental Appendix 10. TransforMED Implementation Index

http://www.annfammed.org/cgi/content/full/8/suppl_1/s9/DC1

Effect of Facilitation on Practice Outcomes in the National Demonstration Project Model

of the Patient-Centered Medical Home

Paul A. Nutting, Benjamin F. Crabtree, Elizabeth E. Stewart; William L. Miller, Ray Palmer, Kurt C. Stange, Carlos Roberto Jaén

Supplemental Appendix 1. National Demonstration Project Model Components

Supplemental Appendix 2. NDP Model Components Implemented by Practices in the Facilitated and Self-Directed Groups

http://www.annfammed.org/cgi/content/full/8/suppl_1/s33/DC1

Journey to the Patient-Centered Medical Home: A Qualitative Analysis of the Experiences of Practices in the National Demonstration Project

Paul A. Nutting, Benjamin F. Crabtree, William L. Miller ${ }_{i}$ Elizabeth E. Stewart, Kurt C. Stange ${ }_{i}$ Carlos Roberto Jaén

Supplemental Appendix. Data Collection, Management, and Analysis

Supplemental Figure. The revised model of practice tested in the NDP, as formalized in 2008.

http://www.annfammed.org/cgi/content/full/8/suppl_1/s45/DC1

Primary Care Practice Development: A Relationship-Centered Approach

William L. Miller, Benjamin F. Crabtree, Kurt C. Stange, Paul A. Nutting; Carlos Roberto Jaén

Supplemental Appendix. Overview of Complexity Theory Concepts

http://www.annfammed.org/cgi/content/full/8/suppl_1/s68/DC1 